\title{
Biodegradation of the diazo dye Reactive Black 5 by a wild isolate of Candida oleophila
}

\author{
Marco S. Lucas ${ }^{\mathrm{a}, \mathrm{b}}$, Carla Amaral ${ }^{\mathrm{a}}$, Ana Sampaio ${ }^{\mathrm{a}}$, José A. Peres ${ }^{\mathrm{b}}$, Albino A. Dias ${ }^{\mathrm{a}, *}$ \\ ${ }^{a}$ CETAV, Departamento de Engenharia Biológica e Ambiental, Universidade de Trás-os-Montes e Alto Douro, Apartado 1013, 5000-911 Vila Real, Portugal \\ ${ }^{\mathrm{b}}$ Centro de Química, Departamento de Química, Universidade de Trás-os-Montes e Alto Douro, Apartado 1013, 5000-911 Vila Real, Portugal
}

Received 17 February 2005; received in revised form 23 August 2005; accepted 14 September 2005

\begin{abstract}
This work looks for a better understanding of the biodegradation of xenobiotic azo dyes mediated by yeasts. During a screening program of phenolic acid assimilating capacities it was found that a non-conventional ascomycetous yeast isolate, identified as Candida oleophila, efficiently decolorizes agar plates supplemented with the commercial textile diazo dye Reactive Black 5. Aerobic batch cultures of C. oleophila could completely decolorize up to $200 \mathrm{mg}$ dye $\mathrm{l}^{-1}$, an ability not yet reported for this yeast species. Moreover, this performance has been achieved in just $24 \mathrm{~h}$ of incubation at $26^{\circ} \mathrm{C}$ in the presence of as little as $5 \mathrm{~g}$ glucose $1^{-1}$ and without visible signs of dye adsorption to yeast cells. It was found that decolorization occurs during the exponential growth phase and neither laccase nor manganese-dependent peroxidase activities were detected in the culture medium. As far as the decolorization mechanism is concerned, our results indirectly suggest the involvement of an azoreductase-like activity in azo bonds cleavage.
\end{abstract}

(C) 2005 Elsevier Inc. All rights reserved.

Keywords: Azo dyes; Bioremediation; Decolorization; Wastewater; Yeasts

\section{Introduction}

Azo dyes are synthetic organic compounds widely used in textile dyeing, paper printing and other industrial processes such as the manufacture of pharmaceutical drugs, toys and foods including candies. This chemical class of dyes, which is characterized by the presence of at least one azo bond $(-\mathrm{N}=\mathrm{N}-)$ bearing aromatic rings, dominates the worldwide market of dyestuffs with a share of about $70 \%$ [1].

Reactive azo dyes released from textile dyeing plants are highly recalcitrant to conventional wastewater treatment processes. In fact, as much as $90 \%$ of reactive dyes could remain unaffected after activated sludge treatment [2]. Therefore, alternative methods should be implemented for effective pollution abatement of dyed effluents. Besides its public acceptance, bioremediation also can be technically attractive since available physical-chemical wastewater treatment processes (e.g. adsorption, filtration, coagulation-flocculation) present some operational problems and high-costs [3,4]. Since 1990, several reports

\footnotetext{
* Corresponding author. Tel.: +351 259 350725; fax: +351 259350266.

E-mail address: jdias@utad.pt (A.A. Dias).
}

have clearly demonstrated the effectiveness of decolorization and dye removal mediated by white-rot basidiomycetous strains belonging to the genera Phanerochaete, Bjerkandera, Phlebia, Pleurotus, Pycnoporus and Trametes among others [5-9]. Fungal treatment of dyed effluents removes several chromophoric groups and thus decreases its toxicity and aesthetic impact in the receiving water bodies. However, much less work was devoted to the decolorization ability of yeasts [10], despite the obvious fact that they are also fungi. From the technological point of view, the development and maintenance of continuous stirred tank reactors operating with unicellular microorganisms, such as yeasts, is a relatively easy task. In fact, filamentous fungi are poorly adapted to a continuous wastewater treatment unit because an exuberant mycelium growth generally occurs $[11,12]$. Previous studies on yeast-mediated color removal (compiled by Fu and Viraraghavan [13]) revealed that azo dye adsorption to biomass was the major decolorization mechanism. More recently and according to the best of our knowledge, it was observed that few ascomycetous yeast species such as Candida zeylanoides [14,15], C. tropicalis, Debaryomyces polymorphus [16] and Issatchenkia occidentalis [17] perform a putative enzymatic biodegradation and concomitant decolorization of azo dyes. 
During a screening program for yeast isolates displaying high phenolic acid removal ability, it was observed that some of them also decolorized the diazo dye Reactive Black 5. Using molecular methods, the most promising isolate was unequivocally identified as a strain of Candida oleophila Montrocher. Interestingly, this species is the biological agent of the product "Aspire" currently used for the post-harvest biocontrol of fruit decay (mostly citrines and pomes) caused by Penicillium sp. and other molds [18]. In this work and for the first time, we report a new ability exhibited by growing cultures of a $C$. oleophila wild strain. Additionally, its performance during batch biodegradation of the industrially important diazo dye Reactive Black 5 as well as the decolorization mechanism is discussed.

\section{Materials and methods}

\subsection{Reagents, dyestuff and microbiological media}

Protocatechuic acid and $p$-hydroxybenzoic acid were obtained from Fluka and Merck, respectively. Caffeic acid, $p$-coumaric acid, syringic acid and 2,2'-azinobis(3-ethylbenzthiazoline-6-sulfonate) (ABTS) were purchased from Sigma-Aldrich. The textile diazo dyestuff Reactive Black 5 (C.I. 20,505) was kindly provided by DyStar Anilinas Texteis Lda (Portugal) and used without prior purification. Yeast malt agar (YM agar) and yeast nitrogen base (YNB) was obtained from Difco. Other chemicals and medium components were at least analytical grade reagents.

\subsection{Screening and yeast isolates maintenance}

Several yeast strains were isolated from the wash water of olives (collected at CAOM, olive oil extraction plant located in Murça, Northern Portugal), by using the spread-plate method carried out on YM agar. Purified yeast isolates were first screened for their ability to grow in the presence of phenolic acids as sole carbon source, using the YNB medium broth supplemented with one of the following substrates: caffeic, protocatechuic, $p$-coumaric, $p$ hydroxybenzoic $\left(1000 \mathrm{mg}^{-1}\right)$, and syringic $\left(100 \mathrm{mg}^{-1}\right)$ acids. Phenolic acid assimilating yeasts were then inoculated into YM agar plates supplemented with the diazo dyestuff Reactive Black $5\left(100 \mathrm{mg} \mathrm{l}^{-1}\right)$ and incubated at $25^{\circ} \mathrm{C}$. Positive isolates were maintained on YM agar slants at $4{ }^{\circ} \mathrm{C}$ and periodically subcultured. The yeast isolate (M33), which exhibited the best performance both on the ability of phenolic acid assimilation and dye decolorization was further identified.

\subsection{Yeast identification}

Total DNA from isolate M33 was extracted using an existing protocol [19] and its modifications [20] after culture growth on MYP agar (malt extract $0.7 \%$, $\mathrm{w} / \mathrm{v}$; yeast extract $0.05 \%$, w/v; soytone $0.25 \%$, w/v; and agar $1.5 \%$, w/v). DNA was amplified using both universal fungal rDNA primers ITS5 (5'-GGA AGT AAA AGT CGT AAC AAG G-3') and LR6 (5'-CGC CAG TTC TGC TTA CC- $3^{\prime}$ ). Cycle sequencing of the D1/D2 domain (a fragment of 600-650 base pairs at the $5^{\prime}$-end of the 26S rDNA) employed forward primer NL1 (5'-GCA TAT CAA TAA GCG GAG GAA AAG-3') and reverse primer NL4 (5'- GGT CCG TGT TTC AAG ACG G-3'). Sequences were obtained with an Amersham Pharmacia ALF express II automated sequencer by Biopremier, Portugal. The obtained sequences were compared with those deposited at the GenBank database (National Center for Biotechnology Information, NCBI) and identified using the Basic Local Alignment Search Tool (BLAST) also available in NCBI.

\subsection{Yeast growth and decolorization of Reactive Black 5}

Batch cultures for growth and biodegradation experiments with the isolate M33 were conducted in minimal medium which contained the following (per litre): $5.0 \mathrm{~g}$ glucose, $1.0 \mathrm{~g}\left(\mathrm{NH}_{4}\right)_{2} \mathrm{SO}_{4}, 1.0 \mathrm{~g} \mathrm{KH}_{2} \mathrm{PO}_{4}, 0.5 \mathrm{~g} \mathrm{MgSO}_{4} \cdot 7 \mathrm{H}_{2} \mathrm{O}, 0.1 \mathrm{~g}$ yeast extract, $0.1 \mathrm{~g} \mathrm{CaCl}_{2} \cdot 2 \mathrm{H}_{2} \mathrm{O}$ and different dyestuff amounts $(0,50,100$, 200, 300, $500 \mathrm{mg}^{-1}$ final concentrations). The minimal medium and dyestuff solutions were autoclaved separately at $121^{\circ} \mathrm{C}$ for $15 \mathrm{~min}$. One $\mathrm{ml}$ of yeast suspension prepared from a recent grown culture in the same medium without dye and aseptically adjusted to an $\mathrm{Abs}_{640}=1.0$ was used to inoculate 250$\mathrm{ml}$ Erlenmeyer flasks containing $100 \mathrm{ml}$ of medium. Incubations were carried out on an orbital shaker set at $120 \mathrm{rpm}$ and $26^{\circ} \mathrm{C}$. Abiotic controls (without yeast inoculation) were always included. Samples were periodically collected for determination of yeast growth, glucose depletion, enzymatic activities and color removal.

\subsection{Other analytical procedures}

Yeast biomass dry weight was determined by the gravimetric method after drying at $100^{\circ} \mathrm{C}$ a three-fold washed yeast suspension until constant weight. Also, a calibration curve was established between biomass dry weight and culture turbidimetry evaluated by absorbance readings at $640 \mathrm{~nm}$. Glucose depletion was determined by the dinitrosalicylic acid reagent [21] for reducing sugars. Culture samples were centrifuged $(7500 \times g, 10 \mathrm{~min})$ and desalted by ultrafiltration before using it for enzymatic assays. The ligninolytic extracellular enzyme activities laccase (EC 1.10.3.2) and manganese-dependent peroxidase (EC 1.11.1.13) were determined as previously reported [22] using $0.1-0.4 \mathrm{ml}$ of culture samples and the respective buffered substrate in $1.5 \mathrm{ml}$ total reaction volume at $25^{\circ} \mathrm{C}$. Briefly, laccase was measured following the oxidation of $2 \mathrm{mM} \mathrm{ABTS}$ at $420 \mathrm{~nm}$. Manganese-dependent peroxidase was measured following the formation of $\mathrm{Mn}$ (III)-tartrate complex at $238 \mathrm{~nm}$. Color removal was determined in centrifuged $(7500 \times g, 10 \mathrm{~min})$ culture samples appropriately diluted with $100 \mathrm{mM}$ phosphate-citrate buffer $\mathrm{pH}$ 7.6. Both absorbance readings at visible maximum peak $(595 \mathrm{~nm})$ and scanning the $\mathrm{UV}$-vis spectrum between 200-800 nm were performed using a Jasco V-530 double-beam spectrophotometer.

All values and data points presented are the means of at least three independent assays unless otherwise stated. The observed standard deviation of experimental data was always less than $7 \%$ of the reported value.

\section{Results and discussion}

\subsection{Properties and identification of a dye-decolorizing yeast strain}

When cultivated on YM agar isolate M33 developed cream colored colonies. It showed the capacity of assimilating as sole carbon and energy source some phenolcarboxylic acids such as, caffeic, protocatechuic, $p$-coumaric, $p$-hydroxybenzoic and syringic. Also, this yeast strain promoted full decolorization zones around colonies grown on YM agar plates containing $100 \mathrm{mg}$ of the diazo dye Reactive Black $51^{-1}$ (Fig. 1) without visible color absorption to the biomass.

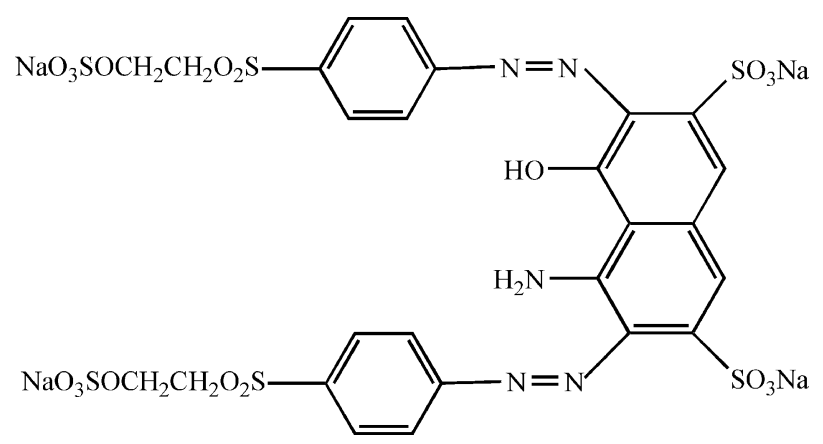

Fig. 1. Chemical structure of the diazo dye Reactive Black 5. 


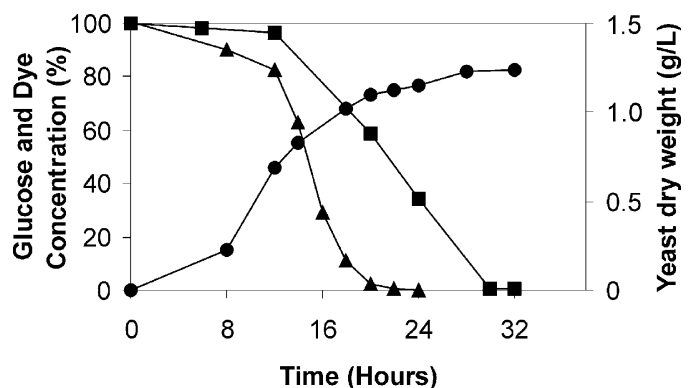

Fig. 2. Time course evolution of yeast growth (-), glucose depletion (ם) and Reactive Black 5 color removal $(\boldsymbol{\Lambda})$ determined in batch cultures of Candida oleophila at an initial dyestuff concentration of $100 \mathrm{mg} / \mathrm{l}$.

The sequence analysis of 26S rDNA D1/D2 domain showed a $100 \%$ homology to the type-strains sequences available on GenBank database. Therefore, ascomycetous strain M33 was identified to the species level as $C$. oleophila Montrocher.

\subsection{Decolorization kinetics of Reactive Black 5 under batch conditions}

In Fig. 2 a typical time course evolution of yeast biomass growth, glucose depletion and decolorization observed in dyecontaining batch cultures of $C$. oleophila is shown. During the trophophase yeast cells depleted both glucose and color at a roughly constant rate and the beginning of exponential yeast growth coincides with the onset of decolorization. Moreover, an apparent close association between glucose depletion and color removal over time was evident. Thus, in this study, we found that growing $C$. oleophila yeast cells decolorize the dye Reactive Black 5 during primary metabolism as previously observed for other ascomycetous yeast species (C. tropicalis and D. polymorphus) [16]. Also, identical behaviour has been observed during the decolorization of different azo dyes by growing cells of the yeast species C. zeylanoides [14] and I. occidentalis [17]. From the practical point of view, and taking into account that dye decolorization is associated to primary metabolism, yeast should be kept in its exponential growth phase so that the bioremediation process occurs much more quickly. On the contrary, filamentous fungi especially of white-rot type typically remove azo dyes during secondary metabolism $[4,8,22,23]$, which implies long incubations periods (usually several days).

Extensive decolorization (95-100\%) was observed in batch cultures containing 50, 100 and $200 \mathrm{mg}$ of Reactive Black $5 \mathrm{l}^{-1}$ (Fig. 3). It is worth noting that C. oleophila achieves this performance within $20-24 \mathrm{~h}$ in the presence of low glucose concentration $(0.5 \%, \mathrm{w} / \mathrm{v})$ and without visible signs of dyestuff adsorption to yeast cells. Since preliminary assays revealed that yeast cells do not grow without glucose, culture medium supplementation with an easily metabolizable carbon and energy source is required for the decolorization process. However, preliminary assays also revealed that a phenolic effluent (olive oil mill wastewater) can be used instead of glucose. Probably, assimilation of a carbon source and its subsequent metabolism through glycolysis, tricarboxylic acids cycle and pentose phosphate pathway supplies yeast cells with reducing power (NADH and/or

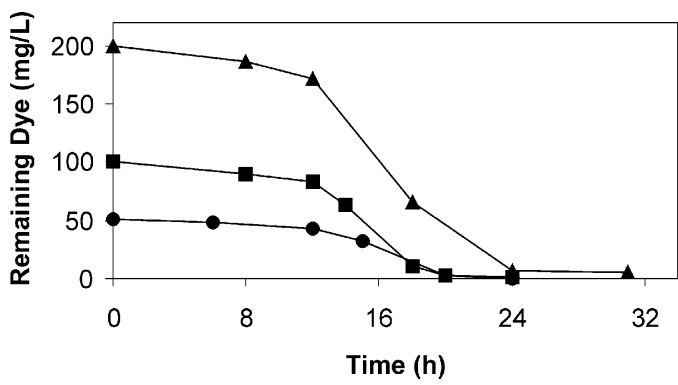

Fig. 3. Time course decolorization of Reactive Black 5 at $50 \mathrm{mg} / \mathrm{l}(\mathbf{}), 100 \mathrm{mg} / \mathrm{l}$ (D) and $200 \mathrm{mg} / 1$ ( $\mathbf{\Lambda})$ initial concentrations in batch cultures of $C$. oleophila.

$\mathrm{FADH}_{2}$ ) required for the reduction of dyestuff azo bonds [17,24]. As far as the decolorization is concerned, growing cultures of $C$. oleophila seems to exhibit an optimum ratio between the initial dyestuff concentration and biomass (dry weight) accumulated after $24 \mathrm{~h}$ of incubation (Fig. 4). At an initial dye concentration of $200 \mathrm{mg} \mathrm{l}^{-1}$ full decolorization occurs which corresponds to yields of $180 \mathrm{mg}$ decolorized dye $\mathrm{g}^{-1}$ biomass (dry weight) and $40 \mathrm{mg}$ decolorized dye $\mathrm{g}^{-1}$ glucose. These yields are substantially higher than previously reported for Reactive Black 5 decolorization by another yeast growing cultures [16]. Since higher initial dye concentrations ( 300 and $500 \mathrm{mg} \mathrm{l}^{-1}$ ) exhibited low decolorization yields but did not significantly affected yeast biomass growth (Fig. 4), an apparent substrate (dye) inhibition occurs. Furthermore, under these conditions yeast cells have a bluish appearance, a phenomenon not observed with batch cultures containing initial dye concentrations up to $200 \mathrm{mg} \mathrm{l}^{-1}$.

\subsection{Decolorization mechanism}

Several spectral scans have been performed during batch decolorization of 50,100 and $200 \mathrm{mg}$ dyestuff $\mathrm{l}^{-1}$ by growing cultures of $C$. oleophila (Fig. 5). Initially, Reactive Black 5 presents two main absorption peaks, one in visible region $(595 \mathrm{~nm})$ and another in UV region $(310 \mathrm{~nm})$, which can be ascribed to the presence of chromophoric azo bonds and both aryl and naphthalene-like moieties, respectively [25]. As it can be seen, significant modification occurred in the dye UV-vis spectrum versus time. Similar decay behaviour of the visible absorption band was also previously observed [16]. The presence of at least one isosbestic point near $300 \mathrm{~nm}$ and a gradual

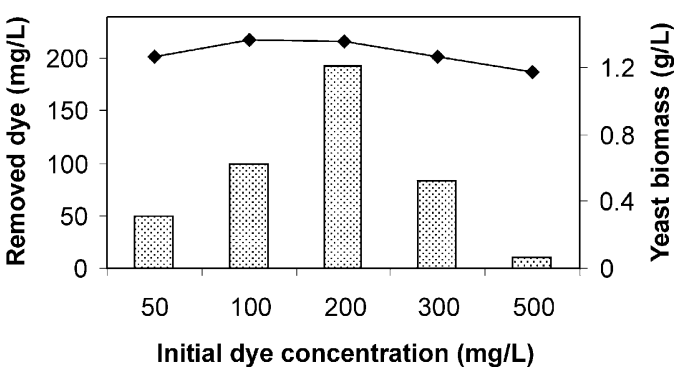

Fig. 4. Effect of different initial dye concentrations in both decolorization (bars) and yeast dry weight accumulation (line) obtained in batch cultures of C. oleophila after $24 \mathrm{~h}$ of incubation. 


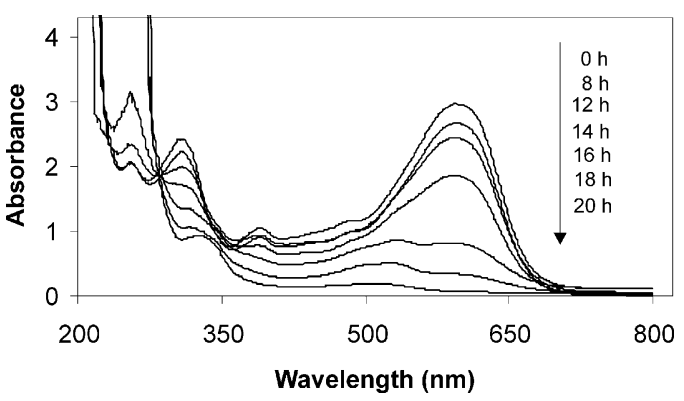

Fig. 5. Example of UV-vis spectral scans from batch cultures of $C$. oleophila containing $100 \mathrm{mg} / \mathrm{l}$ of Reactive Black 5 at different incubation times.

shift of the maximum absorption peak in the visible region from 595 to $525 \mathrm{~nm}$ (Fig. 5), clearly suggests that $C$. oleophila yeast cells decolorized this diazo dye through biodegradation. The fact that batch cultures turned from initial dark-blue to light-pink, which at last tend to colourless and also the absence of visible dyed yeast biomass reinforce this assumption. In order to gain additional insight into the decolorization mechanism, depletion of both color and aromaticity as well as the screening of ligninolytic enzyme activities (laccase and manganese-dependent peroxidase) were also monitored over time. While full color abatement (rupture of chromophoric azo bonds) occurs within $20 \mathrm{~h}$, degradation of aromatic dye moieties proved to be a more recalcitrant process (Fig. 6). Despite manganese-dependent peroxidase might play an important role in the yeast-mediated biodegradation of Reactive Black 5 [16], we have not been able to detect any similar oxidative activity. This is not surprising since the occurrence of ligninolytic enzymes was until now just apparently reported in four yeast species [16,26,27]. It is generally accepted (at least for bacterial processes [3]) but has only recently been proven, that azoreductases play a key role during aerobic azo bond cleavage $[28,29]$ ). In addition, recent physiological and kinetic data strongly suggest that the in vivo yeast-mediated biodegradation of azo dyes [17] also could be ascribable to the activity of a putative aerobic azoreductase. In this work, the observed growth-associated decolorization and concomitant absence of extracellular oxidative enzymes in batch cultures of $C$. oleophila is consistent with an azoreductasecatalysed cleavage of azo bonds previously postulated in the ascomycetous yeast species $C$. zeylanoides [15] and I. occidentalis [17].

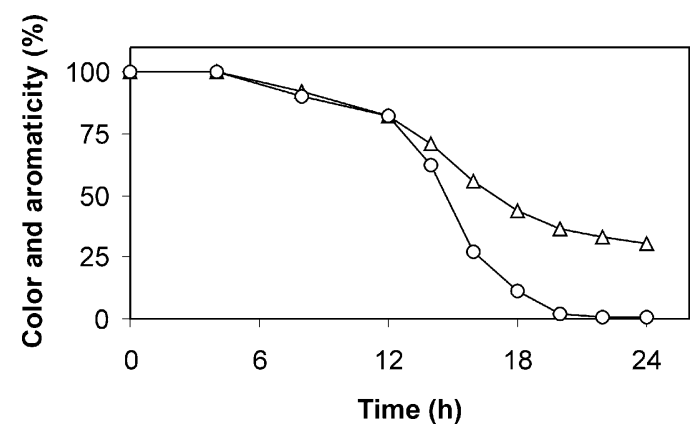

Fig. 6. Evolution of color $(\bigcirc$, absorbance at $595 \mathrm{~nm})$ and aromaticity $(\triangle$, absorbance at $310 \mathrm{~nm}$ ) evaluated in batch cultures of C. oleophila from an initial dyestuff concentration of $100 \mathrm{mg} / \mathrm{l}$.

\section{Conclusions}

An ascomycetous yeast strain isolated from the wash water of olives and identified as C. oleophila efficiently removes the color of the diazo dye Reactive Black 5. Particularly, batch cultures of this yeast species completely decolorize up to $200 \mathrm{mg}_{\text {dye }} \mathrm{l}^{-1}$, within $24 \mathrm{~h}$ of incubation without dye adsorption to the biomass. Also, we do not detect any extracellular ligninolytic activity during the decolorization time course. It was found that azo dye decolorization is associated to primary metabolism and yields of $180 \mathrm{mg}$ decolorized dye $\mathrm{g}^{-1}$ biomass as well as $40 \mathrm{mg}$ decolorized dye $\mathrm{g}^{-1}$ glucose were obtained. Our results, being in line with recent findings, are also consistent with a reductive mechanism of dye decolorization and constitute a new contribution to better understand the potentialities exhibited by non-conventional yeasts on the context of xenobiotic azo dye biodegradation.

\section{References}

[1] Soares GMB, Amorim MTP, Hrdina R, Costa-Ferreira M. Studies on the biotransformation of novel diazo dyes by laccase. Process Biochem 2002;37:581-7.

[2] Pierce J. Colour in textile effluents - the origins of the problem. J Soc Dyers Colourists 1994;110:131-4.

[3] Stolz A. Basic and applied aspects in the microbial degradation of azo dyes. Appl Microbiol Biotechnol 2001;56:69-80.

[4] Wesenberg D, Kyriakides I, Agathos SN. White-rot fungi and their enzymes for the treatment of industrial dye effluents. Biotechnol Adv 2003;22:161-87.

[5] Cripps C, Bumpus JA, Aust SD. Biodegradation of azo and heterocyclic dyes by Phanerochaete chrysosporium. Appl Environ Microbiol 1990;56:1114-8.

[6] Rodríguez E, Pickard MA, Vazquez-Duhalt R. Industrial dye decolorization by laccases from ligninolytic fungi. Curr Microbiol 1999;38:27-32.

[7] Gill PK, Arora DS, Chander M. Biodecolorization of azo and triphenylmethane dyes by Dichomitus squalens and Phlebia spp. J Ind Microbiol Biotechnol 2002;28:201-3.

[8] Selvam K, Swaminathan K, Chae K-S. Microbial decolorization of azo dyes and dye industry effluent by Fomes lividus. World J Microbiol Biotechnol 2003;19:591-3.

[9] Máximo C, Amorim MTP, Costa-Ferreira M. Biotransformation of industrial reactive azo dyes by Geotrichum sp. CCMI 1019. Enz Microbiol Technol 2003;32:145-51.

[10] Forgacs E, Cserháti T, Oros G. Removal of synthetic dyes from wastewaters: a review. Environ Int 2004;30:953-71.

[11] Assas N, Marouani L, Hamdi M. Scale down and optimization of olive mill wastewaters decolorization by Geotrichum candidum. Bioprocess Eng 2000;22:503-7.

[12] López C, Mielgo I, Moreira MT, Feijoo G, Lema JM. Enzymatic membrane reactors for biodegradation of recalcitrant compounds. Application to dye decolourization. J Biotechnol 2002;99:249-57.

[13] Fu Y, Viraraghavan T. Fungal decolorization of dye wastewaters: a review. Bioresource Technol 2001;79:251-62.

[14] Martins MAM, Cardoso MH, Queiroz MJ, Ramalho MT, Campos AMO. Biodegradation of azo dyes by the yeast Candida zeylanoides in batch aerated cultures. Chemosphere 1999;38:2455-60.

[15] Ramalho PA, Scholze H, Cardoso MH, Ramalho MT, OliveiraCampos AM. Improved conditions for the aerobic reductive decolourization of azo dyes by Candida zeylanoides. Enz Microbiol Technol 2002;31:848-54.

[16] Yang Q, Yang M, Pritsch K, Yediler A, Hagn A, Schloter M, et al. Decolorization of synthetic dyes and production of manganese-dependent peroxidase by new fungal isolates. Biotechnol Lett 2003;25:709-13. 
[17] Ramalho PA, Cardoso MH, Cavaco-Paulo A, Ramalho MT. Characterization of azo reduction activity in a novel ascomycete yeast strain. Appl Environ Microbiol 2004;70:2279-88.

[18] Mari M, Bertolini P, Pratella GC. Non-conventional methods for the control of post-harvest pear diseases. J Appl Microbiol 2003;94: 761-6.

[19] Sampaio JP, Gadanho M, Bauer R. Taxonomic studies on the genus Cystofilobasidium: description of Cystofilobasidium ferigula sp. nov and clarification of the status of Cystofilobasidium lari-marini. Int J Sys Evol Microbiol 2001;51:221-9.

[20] Gadanho M, Almeida JMGCF, Sampaio JP. Assessment of yeast diversity in a marine environment in the south of Portugal by microsatelliteprimed PCR. Anton Leeuw Int J G 2003;84:217-27.

[21] Miller GL. Use of dinitrosalicylic acid reagent for determination of reducing sugar. Anal Chem 1959;31:426-8.

[22] Dias AA, Bezerra RM, Lemos PM, Pereira AN. In vivo and laccasecatalysed decolourization of xenobiotic azo dyes by a basidiomycetous fungus: characterization of its ligninolytic system. World J Microbiol Biotechnol 2003;19:969-75.
[23] Kirby N, Marchant R, McMullan G. Decolourization of synthetic textile dyes by Phlebia tremellosa. FEMS Microbiol Lett 2000;188:93-6.

[24] Gottlieb A, Shaw C, Smith A, Wheatley A, Forsythe S. The toxicity of textile reactive azo dyes after hydrolysis and decolourization. J Biotechnol 2003;101:49-56.

[25] Silverstein RMC, Bassler GC, Morrill TC. Spectrophotometric identification of organic compounds. New York: John Wiley and Sons; 1991.

[26] Villas-Boas SG, Esposito E, Mendonça MM. Novel lignocellulolytic ability of Candida utilis during solid-substrate cultivation on apple pomace. World J Microbiol Biotechnol 2002;18:541-5.

[27] Zhu XD, Williamson PR. Role of laccase in the biology and virulence of Cryptococcus neoformans. FEMS Yeast Res 2004;5:1-10.

[28] Chen HZ, Wang RF, Cerniglia CE. Molecular cloning, overexpression, purification, and characterization of an aerobic FMNdependent azoreductase from Enterococcus faecalis. Protein Express Purif 2004;34:302-10.

[29] Nachiyar CV, Rajakumar GS. Purification and characterization of an oxygen insensitive azoreductase from Pseudomonas aeruginosa. Enz Microbiol Technol 2005;36:503-9. 